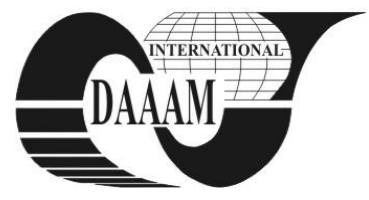

Annals of DAAAM for 2011 \& Proceedings of the 22nd International DAAAM Symposium, Volume 22, No. 1, ISSN $1726-9679$ ISBN 978-3-901509-83-4, Editor B. Katalinic, Published by DAAAM International, Vienna, Austria, EU, 2011 Make Harmony between Technology and Nature, and Your Mind will Fly Free as a Bird Annals \& Proceedings of DAAAM International 2011

\title{
A NUMERICAL METHOD FOR ALMOST INCOMPRESSIBLE BODY PROBLEM
}

\author{
BIJELONJA, I[zet]
}

\begin{abstract}
This paper describes development of a displacement/pressure finite volume based method for modeling of compressible and incompressible linear elastic body problem. The finite volume approach is used to solve a steady case problem of an epoxy disc with an inserted a crack and enclosed in a thin steel ring. The numerical method is based on the solution of the integral form of conservation equations governing momentum balance. A segregated approach is employed to solve resulting set of coupled linear algebraic equations, embedding a SIMPLE based algorithm for displacement-pressure coupling. Numerical results show that applied numerical method appears to be locking and pressure oscillations free.
\end{abstract}

Key words: numerical analysis, finite volume method, almost incompressible body, displacement, pressure formulation

\section{INTRODUCTION}

An analysis of deformation of almost incompressible solids is related to rubber-like materials, some elastomers and materials in inelastic condition.

Numerical analysis of deformation of solids in incompressible limit could lead to volumetric locking phenomenon and pressure oscillation. To overcome the problems a number of approaches have been proposed under the finite element procedures (Bathe, 1996; Belytschko \& Dolbow, 1999.).

The interest for finite volume (FV) application to the structural analysis problems involving incompressible materials has grown recently (Wheel, 1999). The model developed for the analysis of incompressible fluids (Demirdžić \& Muzaferija, 1995) is adopted in (Bijelonja at al., 2006.) to describe, in a displacement/pressure based formulation, deformation of an incompressible elastic solid. Numerical experiments showed that developed discretisation scheme is free of pressure oscillation and volumetric locking for finite volume meshes of arbitrary topology.

In this paper, a finite volume based (displacement/pressure) formulation, which is valid for linear elastic compressible as well as fully incompressible solid, is presented. The method is based on the solution of the integral form of momentum balance equation. Constitutive equations, which are valid for both compressible and incompressible linear elastic materials, are employed. A collocated variable arrangement is used, and a segregated approach is employed to solve resulting set of coupled linear algebraic equations.

The method is applied to a practical case of an epoxy (almost incompressible) disc with an inserted crack which is enclosed in a thin steel (compressible) ring. The FV method results are compared with the finite element solution.

In the next section the governing equations together with constitutive relations are given. This is followed by a brief description of the finite volume discretisation procedure. Finally, the method's capabilities are demonstrated by applying it to a compound body made of an epoxy disc enclosed in a thin steel ring.

\section{MATHEMATICAL FORMULATION AND NUMERICAL DISCRETIZATION}

The behavior of a continuum is governed by the following momentum transport equation:

$$
\frac{\partial}{\partial t} \int_{V} \rho \frac{\partial \mathbf{u}}{\partial t} \mathrm{~d} V=\int_{S} \sigma \cdot \mathrm{d} \mathbf{s}+\int_{V} \rho \mathbf{f}_{\mathrm{b}} \mathrm{d} V,
$$

which is valid for an arbitrary part of continuum of the volume $V$ bounded by the outward pointing surface vector $\mathbf{s}$. In equation (1), $\rho$ is the mass density, $\mathbf{u}$ is the displacement vector, $\boldsymbol{\sigma}$ is the Cauchy stress tensor, and $\mathbf{f}_{\mathrm{b}}$ is the resulting body force. It is assumed here that angular momentum balance equation is satisfied exactly due the shear stresses conjugate principle.

The constitutive equations for an isotropic linear elastic solid may be written as a pair of the following equations:

$$
\begin{aligned}
& \sigma=2 \mu \varepsilon-p \mathbf{I}, \\
& \frac{p}{\lambda}+\operatorname{div} \mathbf{u}=0,
\end{aligned}
$$

where $\boldsymbol{\varepsilon}$ is the linear strain tensor, $p$ is the pressure parameter, $\mathbf{I}$ is the identity tensor, $\lambda$ and $\mu$ are the Lame constants, related to the Young's modulus $E$ and the Poisson's ratio $v$.

Integrating constitutive equation (3) and using the Gauss divergence theorem the following equation is obtained:

$$
\int_{V} \frac{p}{\lambda} \mathrm{d} V+\int_{S} \mathbf{u} \cdot \mathrm{d} \mathbf{s}=0
$$

Introducing constitutive relation (2) into momentum balance equation (1), equations (1) and (4) make a closed set of four equations with four unknown functions (three displacement vector components $u_{\mathrm{i}}$ and pressure $p$. These equations are discretised by employing the finite volume procedures described in (Demirdžić \& Muzaferija, 1994; Demirdžić \& Muzaferija, 1995).

In order to obtain discrete counterpart of equations (1) and (4) in a steady case, the space is discretised by a number of contiguous, non-overlapping control volumes (Fig. 1), with computational points at their centers.

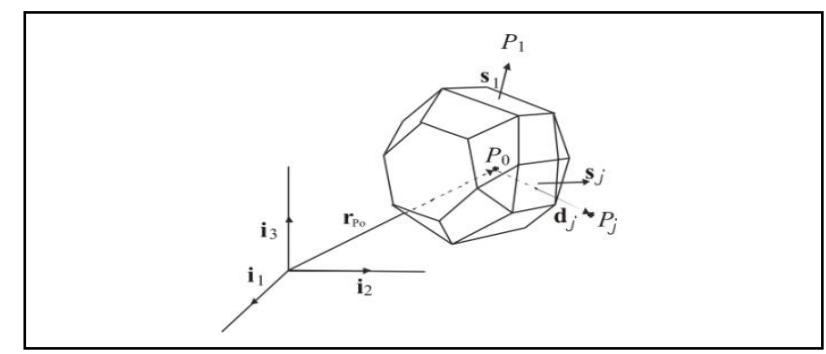

Fig.1. A control volume of an arbitrary shape 
After the space discretisation, equations (1) and (4) are written for each control volume. For example, in the case of momentum equation follows:

$$
\begin{aligned}
\frac{\partial}{\partial t} \int_{V} \rho \frac{\partial u_{i}}{\partial t} \mathrm{~d} V & =\sum_{j=1}^{n_{f}} \int_{S} \mu \operatorname{grad} u_{i} \cdot \mathrm{d} \mathbf{s}+ \\
& \sum_{\mathrm{j}=1}^{\mathrm{n}_{f}} \int_{S}\left[\mu\left(\operatorname{grad} u_{i}\right) \cdot \mathbf{i}_{\mathrm{i}}-p \mathbf{i}_{\mathrm{i}}\right] \cdot \mathrm{d} \mathbf{s}+\int_{V} \rho f_{b_{i}} \mathrm{~d} V,
\end{aligned}
$$

where $\mathbf{i}_{\mathrm{i}}$ are the Cartesian base vectors and $n_{\mathrm{f}}$ is the number of faces enclosing the cell $P_{0}$. In numerical discretisation of governing equations linear distribution of dependent variables is assumed between computation points, and the surface and volume integrals are calculated using the midpoint rule. A segregated approach is employed to solve resulting set of coupled linear algebraic equations, embedding a SIMPLE based algorithm (Patankar, 1980) for calculation of the pressure parameter featuring in the constitutive equation.

\section{STUDY CASE}

An epoxy disc enclosed in a thin steel ring and with a star shaped hole consisting six symmetrically located leaflets is considered (Fig. 2). In the epoxy, $25.4 \mathrm{~mm}$ long cracks in the radial direction at the tip of the leaflets are inserted. A plane strain deformation of the composite cylindrical body under a uniform pressure of $6.894 \mathrm{MPa}$ inside the star shaped hole is studied in (Batra, 2002) using finite element method by the commercial code ABAQUS 6.11. The space domain is divided into 4824 eight node element with biquadratic interpolation for displacement and linear interpolation for the pressure field. The epoxy and the steel casing are modelled as isotropic and homogeneous materials.

Deformation of the steel ring are to be infinitesimal and its material is modelled by Hooke's law with Young's modulus $E=$ $2 \cdot 10^{5} \mathrm{MPa}$ and Poisson's ratio $v=0.3$. In finite element calculation epoxy is modelled either as a Mooney-Rivlin or as a Hookean material. In a case of the Hookean material the Poisson's ratio varied from 0.49 to 0.4999 and the shear modulus equals to $10.19 \mathrm{MPa}$.

The finite volume calculations are performed using the mesh, locally refined around the leaflet corner and the the crack, consisting of 2844 control volumes, as shown in Fig. 2 (right). Because of the symmetry of the problem, only a $30^{\circ}$ segment of the body is investigated. In planes $\theta=0^{0}$ and $\theta=$ $30^{\circ}$ the symmetry plane boundary conditions are imposed, and the outer surface of the steel ring is taken to be traction free. The epoxy disc is assumed to be perfectly bonded to the steel ring so that the displacement and the surface traction are continuous across their common interface.

Deformed shapes of the crack face calculated by finite element and finite volume method are plotted in Fig. 3. The numerical results are shown for two different Poisson's ratios, 0.49 and 0.4999 . It can be seen that crack opening displacements decrease with an increase in Poisson's ratio and

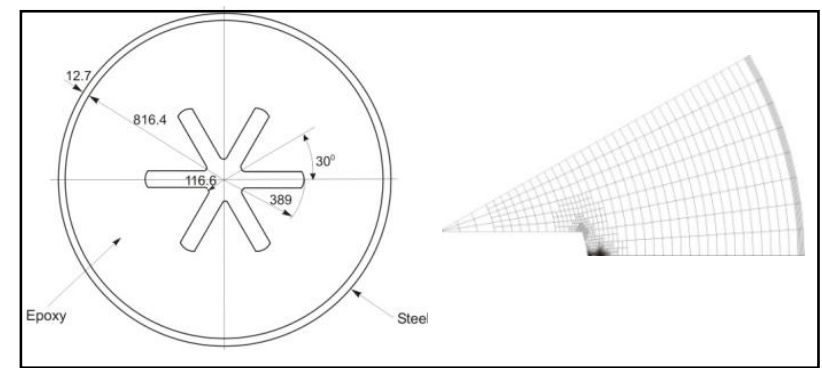

Fig. 2. A cross-section of the epoxy disc enclosed in a thin steel ring (dimensions in millimeters) (left) and computational mesh (right)

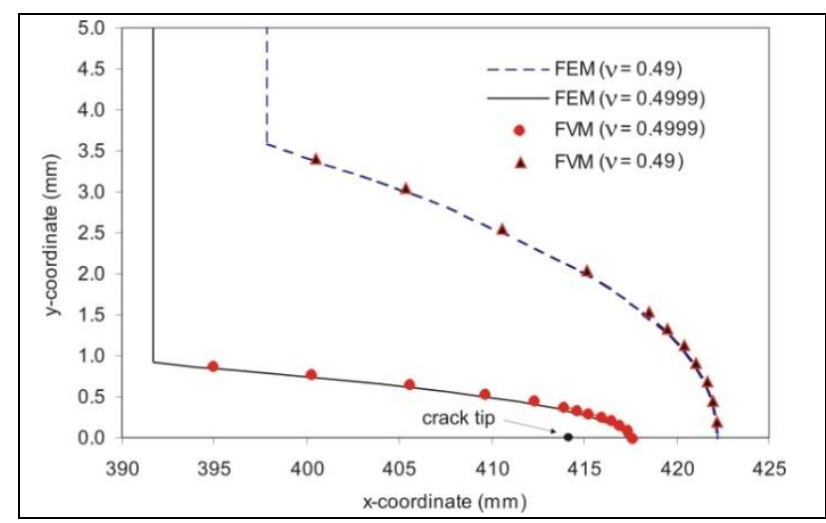

Fig.3. Deformed shapes of the crack face

that curvature of the deformed crack at the tip increases with an increase in Poisson's ratio. Finite volume calculations agree very well with finite element results.

\section{CONCLUSION}

In this paper a displacement/pressure finite volume based numerical procedures for predicting the linear elastic behavior of compressible as well as incompressible elastic solid is successfully applied. The application of the numerical procedures on presented steady case demonstrates very good accuracy of the method.

The volumetric locking phenomenon, common in numerical description of an incompressible and nearly incompressible material behavior, is not registered. Another phenomenon, nonphysical pressure oscillations, which are also inherent in numerical analysis of incompressible and nearly incompressible body, is effectively eliminated using the SIMPLE based algorithm for displacement-pressure coupling.

The future work will be focused on a generalization of the displacement/pressure finite volume formulation to the unsteady $3 \mathrm{D}$ problems of linear elasticity.

\section{REFERENCES}

Bathe, K.J. (1996). Finite Element Procedures, Prentice-Hall, ISBN 0-13-301458-4, New Jersey

Batra, R.C. \& Ching, H.K. (2002). Energy release rates in a constrained epoxy disc with Hookean and Mooney-Rivlin materials, Theoretical and Applied Fracture Mechanics, Vol.38, (2002) pp. 165 - 175, ISSN 0167-8442

Belytschko, T. \& Dolbow, J. (1999). Volumetric locking in the Element Free Galerkin method, Int. J. Numer. Methods Engrg., Vol.46, (1999) pp. 925 - 942, ISSN 1097-0207

Bijelonja, I.; Demirdžić, I. \& Muzaferija, S. (2006). A finite volume method for incompressible linear elasticity, Comput. Methods Appl. Mech. Engrg., Vol.195, (2006) pp. 6378 - 6390, ISSN 0045-7825

Demirdžić, I. \& Muzaferija , S. (1994). Finite volume method for stress analysis in comlpex domains, Int. J. Numer. Methods Engrg., Vol.37, (1994) pp. 3751 - 3766, ISSN 1097-0207

Demirdžić, I. \& Muzaferija, S. (1995). Numerical method for coupled fluid flow, heat transfer and stress analysis using unstructured moving meshes with cells of arbitrary topology, Comput. Methods Appl. Mech. Engrg., Vol.125, (1995) pp. 235 - 255, ISSN 0045-7825

Patankar, S.V. (1980). Numercal Heat Transfer and Fluid Flow, McGraw-Hill, ISBN 0891165223

Wheel, M.A. (1999). A mixed finite volume formulation for determining the small strain deformation of incompressible materials, Int. J. Numer. Methods Engrg., Vol.44, (1999) pp. 1843 - 1861, ISSN 1097-0207 